\title{
Protective effects of baicalin on lipopolysaccharide (LPS)-induced implantation failure and the uterine endometrium in mice
}

\author{
Yantao Zhao', Yongzhan Bao', Wanyu Shi ${ }^{1,2}$, Xiaodan Wang ${ }^{2}$ and Xiuhui Zhong ${ }^{1,2^{\star}}$ \\ ${ }^{1}$ College of Veterinary Medicine, Agricultural University of Hebei, Baoding 071001, China. \\ ${ }^{2}$ Institute of Traditional Chinese Veterinary Medicine, Agricultural University of Hebei, China.
}

Accepted 4 April, 2011

\begin{abstract}
The objective of this study is to investigate the protective effects of baicalin and to elucidate its immunological modulation at maternal-fetal interface. Lipopolysaccharide (LPS) was given via intraperitoneal injection to induce embryo implantation failure in mice which received baicalin at days 0.5 to 3.5 of gestation. The IFN-y/LL-10, nitric oxide synthase (NOS) activities of uterine and endometrial tissues in each group $(n=16)$ were detected by ELISA, respectively. The ratio of IFN-Y/IL-10 and NOS activities increased significantly in the uterus of LPS-induced mice implantation failure and endometrial cells of LPS interference. In the baicalin pretreated mice followed by LPS administration, the ratio of IFN-y/IL-10 and NOS activities were normalized by the middle dose baicalin to the control level, significantly lower than LPS treatment group. The same results were received in vitro study of LPS interference endometrial cells. The results indicate that baicalin has protective effects through inhibiting excessive IFN- $y$ and NO production to modulate the immunological balance at maternal-fetal interface.
\end{abstract}

Key words: Baicalin, lipopolysaccharide (LPS), implantation failure, IFN-y, IL-10, nitric oxide (NO).

\section{INTRODUCTION}

Pregnancy is a complex, sophisticated physiological process. Embryo implantation is one of the earliest events in reproduction of mammals that determines whether pregnancy will be established successfully. Embryo implantation is an absolute requirement for reproduction. Successful implantation depends on the semiallogenic embryo, tolerant maternal endometrium and their synchronization. The implantation process, being considered as a sequence of biochemical and physical interactions between the embryo and the uterus, primarily begins with an intimate cross-talk between them.

The relative low efficiency of the implantation is paradoxical in view of the fact that reproduction is critical to species survival. Implantation failure remains an unsolved problem in reproduction. Indeed, the average implantation rate in vitro fertilization (IVF) is about $25 \%$ (de los Santos et al., 2003). The main reason for the

*Corresponding author. E-mail: zxh8078@163.com, clock8078@126.com. Fax: 86-312-2381158. implantation failure is inadequate uterine receptivity (Simon et al., 1998; Ledee-Bataille et al., 2002). Implantation involves a complex sequence of signaling events, with many identified molecular mediators, including adhesion molecules, cytokines, growth factors, lipids and others (Lessey et al., 1992; Simon et al., 2000).

The immune balance at the maternal-fetal interface is necessary for the complicated pregnancy establishment and maintenance, especially $T$ helper 1 (Th1)/Th2 cytokine balance. Th1 cytokines, such as interleukin-2 (IL-2), interferon- $\gamma$ (IFN- $\gamma$ ) and tumor necrosis factor- $\alpha$ (TNF- $\alpha$ ) are detrimental to pregnancy. Th2 cytokines are assumed to be a pregnancy-preferred Th2-bias. So a significantly increased Th1 cytokine expression may be the main reason for pregnancy failures (Krasnow et al., 1996; Kalinkaand Radwan, 2006).

Nitric oxide (NO) is now recognized as an important mediator of functions in a variety of physiological systems (Moncada et al., 1992), including the reproductive system (Chatterjee et al., 1996; Yallampalli et al., 1998). Endogenous NO is synthesized by NO synthase (NOS) (Moncada et al., 1992). NO may mediate the embryo implantation in normal physiological situation. Though, 
embryo implantation is unsuccessful with blocking NO production, excessive NO for a long duration may make endometrium injured (Knowles and Moncada, 2002; Wu et al., 2003).

Baicalin, a type of flavonoid, is one of the major components of Chinese medicinal herb Huangqin (Radix scutellariae). Huangqin has an anti-abortive effect through inhibiting natural killer (NK) cell infiltration and regulating the cytokines at the maternal-fetal interface (Zhong et al., 2002, 2008). However, whether baicalin has an anti-abortive effect and how it works are less documented. In the present study, we used lipopolysaccharide (LPS) to induce the embryo implantation failure model, and measured IFN- $y$, IL-10 levels and NOS activity with an aim to elucidate the protective effects of baicalin on the abortion model and cytokines at the maternal-fetal interface.

\section{MATERIALS AND METHODS}

\section{Preparation of reagents}

LPS (Sigma Chemicals) from Escherichia coli was dissolved in sterile saline at a final concentration of $0.04 \mathrm{mg} / \mathrm{ml}$. Baicalin (National Institutes of Food and Drug Control, China) was dissolved with distilled water, the final concentrations being $0.25,1.25$ and 2.5 $\mathrm{mg} / \mathrm{ml}$, respectively.

\section{Treatment of animals}

Kunming species virgin mice (body weight $25 \pm 2 \mathrm{~g}$ ) aged 7 to 8 weeks were purchased from the Laboratory Animal Center of Hebei Medical University, China. Before the study, they were acclimatized for one week in the laboratory under standard conditions of temperature $\left(23 \pm 2^{\circ} \mathrm{C}\right)$ and illumination ( $12 \mathrm{~h}$ dark: $12 \mathrm{~h}$ light) cycle, and were given free access to mouse chow and water. The animals were mated by housing one female with one male and the females were examined each day in the early morning. Pregnancies were obtained for the presence of a vaginal plug. The day the vaginal plug was detected was designated as day 0.5 of pregnancy (Zhang and Zhong, 2010).

The pregnant mice were randomly divided into 5 groups (16 mice/group), including the control group (A), the LPS treatment group $(B)$, the low-dose baicalin group $(C, 0.1 \mathrm{mg})$, the middle-dose baicalin group $(D, 0.5 \mathrm{mg})$ and the high-dose baicalin group $(E, 1.0$ $\mathrm{mg}$ ). Mice in groups $\mathrm{C}, \mathrm{D}$ and $\mathrm{E}$ were given three different dosages of baicalin $0.4 \mathrm{ml}$ per day via oral gavage on gestation days 0.5 to 3.5. Mice in groups $A$ and $B$ received distilled water instead of baicalin by oral gavage at the same volume. Mice in groups $B, C, D$ and $E$ were given via intraperitoneal injection (i.p.) of $0.1 \mathrm{ml}(0.004$ $\mathrm{mg}$ ) LPS per mouse on day 3 of gestation (the dosage derived from the preliminary data, not shown). Animals in group $A$ were administrated with sterile saline at $0 . \mathrm{ml}$ on day 3 of gestation. The mice were sacrificed, respectively on day 4.5 and 6.5 of gestation. Animals were treated according to the Guidelines for Keeping Experimental Animals issued by Chinese government.

\section{Treatments of endometrial cells}

The uterine specimens were removed immediately when the mice were sacrificed by cervical dislocation on day 4.5 of gestation, and then were transported to a sterile laminar flow hood. The specimens were put on the cool sterile frosted glass and fat tissue was gotten rid of, washed in sterile Hank's Balanced Salt Solution (HBSS). Then the uterine tissue was dissected and cut into 0.5 to $1.0 \mathrm{~mm}^{3}$ and digested with $0.25 \%$ trypsin (Beijing Solarbio Science \& Technology Co. Ltd) in the presence of $0.04 \%$ ethylenediaminetetraacetic acid (EDTA) for 10 to $15 \mathrm{~min}$ at $37^{\circ} \mathrm{C}$. The DMEM/F-12 supplemented with $10 \%$ fetal bovine serum (FBS) was added to end the digestion. The digested mixture was filtered through a $74 \mu \mathrm{m}$ cell strainer (200 meshes) to remove undigested tissue fragments. The filtrate was washed 3 to 5 times with culture medium at $1100 \mathrm{r} / \mathrm{min}$ for $5 \mathrm{~min}$. The number of viable cells that excluded Trypan blue was counted using a hemocytometer. After cell counting and viability determination, the endometrial cells were seeded at the rate of $3 \times 10^{5}$ viable cells in DMEM/F-12 containing $10 \%$ FBS in cell culture plates $(6,24$ and 96 well plates) in the presence of $5 \% \mathrm{CO}_{2}$ at $37^{\circ} \mathrm{C}$. The medium was changed according to the situation of cell growth with regular observation.

The cell viability was conducted by 3-(4,5-dimethythiazol-2-yl)2,5-diphenyl tetrazolium bromide (MTT) assay to select the suitable concentrations of LPS and baicalin. From the results of MTT, we got that the LPS optimal concentration was $100 \mathrm{ng} / \mathrm{ml}$ and the baicalin effective concentration were 40,400 and $800 \mu \mathrm{g} / \mathrm{ml}$.

The cells growing well at logarithmic phase in 24-well plate were divided into five groups: Group I was kept as the control (4 wells). Group II was LPS (100 ng/ml) interference group (5 wells). Group III was low-dose baicalin $(40 \mu \mathrm{g} / \mathrm{ml})$ group (5 wells), Group IV as middle-dose baicalin $(400 \mu \mathrm{g} / \mathrm{ml})$ Group (5 wells) and group V as high-dose baicalin $(800 \mu \mathrm{g} / \mathrm{ml})$ group (5 wells). The medium was changed every $24 \mathrm{~h}$ and the cell situation was observed. The cell culture supernatants were collected at $48 \mathrm{~h}$ for enzyme-linked immunosorbant (ELISA) assay.

\section{Calculation of the implanted embryo number and abortion rate}

All gravid female mice were sacrificed by cervical dislocation at day 6.5 of gestation and the contents of uterus were examined for viable and resorbing embryos. The viable embryos were well-oxygenated (pink) and showed a well-defined embryonic capsule and placenta. The resorbing embryos were usually smaller, showed signs of ischemia, hemorrhage and often were macerated and black in color without identifiable embryo or placenta. The incidence of abortion was presented as a percentage of the contents of the miscarriage (100 abortive mice/total mice). The number of implanted embryo was presented as average implanted embryo of each pregnant mouse (total implanted embryos/pregnant mice).

\section{Measurement of IFN-y, IL-10 and NOS activity by ELISA}

All pregnant mice were sacrificed by cervical dislocation before blood samples were harvested in posterior orbital venous plexus approach at day 4.5 of gestation. The left uterine horn used for ELISA was carefully cleaned, with the fetuses removed. Uterine lysates were prepared in PBS ( $\mathrm{pH}$ 7.4) containing phenylmethanesulfanyl fluoride (PMSF, $0.75 \mu \mathrm{g} / \mathrm{ml}$, Sigma) and centrifuged for $15 \mathrm{~min}$ at 12000 $\mathrm{r} / \mathrm{min}$ at $4^{\circ} \mathrm{C}$ and the supernatants were collected for ELISA. The cell culture supernatants were collected at $48 \mathrm{~h}$ for ELISA. IFN-y, IL-10 levels and NOS activity of the uterine lysate and cell culture supernatant were assessed using an IFN- $\gamma$ ELISA kit (R\&D systems, USA) and IL-10 ELISA kit (R\&D systems, USA) and nitric oxide synthase (NOS) detection kit (Nanjing Jiancheng Bioengineering Institute, China), respectively, according to the manufacturer's instructions.

\section{Statistical analysis}

All data are expressed as means \pm SD. Statistical analysis of data 
Table 1. The protective effects of baicalin from LPS-induced embryo implantation failure $(n=8$, unit: individual).

\begin{tabular}{lcc}
\hline Group & Average number of implanted embryo & Abortion rate (\%) \\
\hline A: Control & $8.57 \pm 1.270^{\mathrm{a}}$ & $12.5(1 / 8)$ \\
B: LPS treatment & $2.50 \pm 0.707^{\mathrm{b}}$ & $75(6 / 8)$ \\
C: $0.1 \mathrm{mg}$ baicalin + LPS & $5.75 \pm 0.957^{\mathrm{c}}$ & $50(4 / 8)$ \\
D: $0.5 \mathrm{mg}$ baicalin + LPS & $7.60 \pm 1.140^{\mathrm{ac}}$ & $37.5(3 / 8)$ \\
E: $1.0 \mathrm{mg}$ baicalin + LPS & $7.00 \pm 1.410^{\mathrm{ac}}$ & $25(2 / 8)$ \\
\hline
\end{tabular}

Data with different letters indicate significant differences at $p<0.05$.
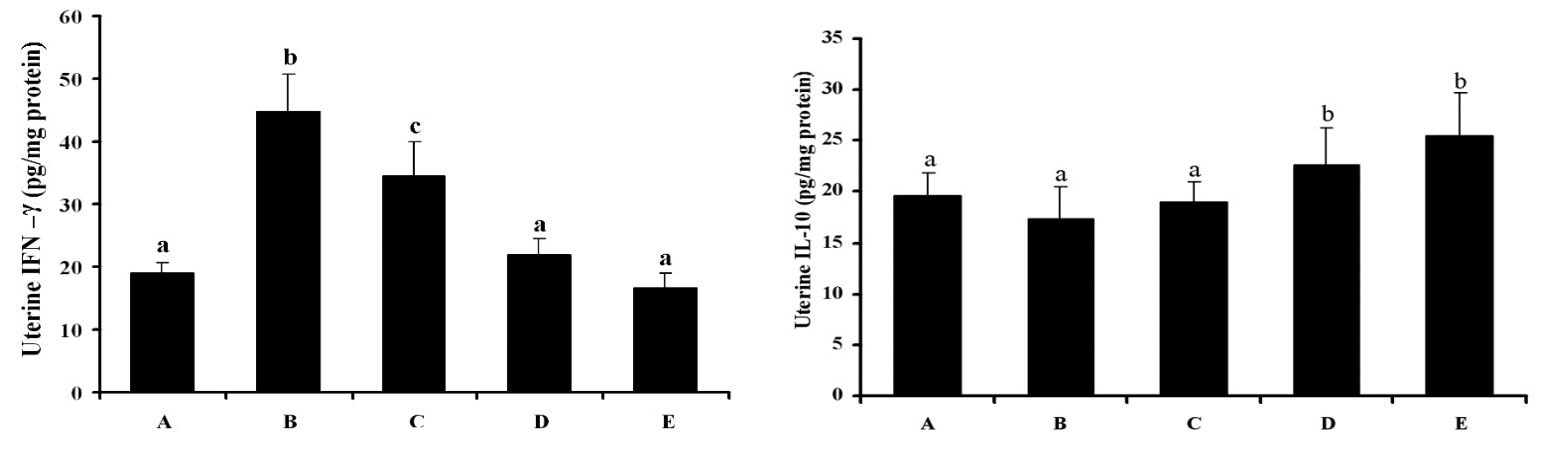

Figure 1. The uterine IFN- $\mathbf{y}$ and IL-10 levels in different treatments mice $(n=8)$. Values of IFN- $\mathbf{y}$ and IL-10 were expressed as $\mathrm{pg} / \mathrm{mg}$ protein. Significant differences $(p<0.05)$ among all the groups were determined by ANOVA and are indicated by different letters.

were conducted using SPSS 13.0, analyzed with one-way analysis of variance (ANOVA). In all statistical tests, $p$ values less than 0.05 were considered as significant.

\section{RESULTS}

\section{Baicalin increases embryo implantation}

The control mice (group A) pretreated with saline showed the average number of implanted embryo was $8.57 \pm$ 1.270 and the natural implantation rate was $80 \%$. The average number of implanted embryo of LPS model group (B) was significantly less than other groups (2.50 \pm $0.707)$. There was no significant difference between the baicalin groups (D and $E$ ) and control, except the lowdose baicalin group $\mathrm{C}$. There was also no difference among the three-dose baicalin groups. However, the implanted embryos of the middle-dose baicalin group (D) were the most. The abortion rate decreased along with the baicalin dosage increasing (Table 1). However, the baicalin with three concentrations dose did not reverse the LPS-induced implantation failure in mice.

\section{Baicalin alters uterine IFN-y and IL-10 levels}

The mice uterine IFN-y levels in group B treated with LPS significantly increased when compared with the control group. However, LPS treatment did not significantly change the uterine IL-10 levels (Figure 1). The IFN-Y levels in baicalin treatment groups ( $D$ and $E$ ) significantly decreased when compared with LPS treatment group, except the low-dose baicalin group $(\mathrm{C})$. Meanwhile, the uterine IL-10 contents in middle and high dose baicalin groups (D and E) significantly increased when compared with control and LPS treatment group. And the uterine IL10 levels increased along with the baicalin dosage from low to high. The ratio of IFN- $y / \mathrm{LL}-10$ was significantly increased with LPS treatment, but it is adjusted to near control level plus baicalin $(0.5 \mathrm{mg})$ administration. The results herein, indicated that baicalin down-regulate LPSinduced IFN- $\gamma$ changes in the uterus. And the baicalin can increase the uterine IL-10 content in mice.

\section{Effects of baicalin on NOS activities of uterus tissue and serum}

The uterine NOS activity in LPS treatment group significantly increased when compared with the control group (Figure 2) and uterine NOS activity was significantly decreased by baicalin when compared with LPS treatment group. Uterine NOS activities of the middle and high dose baicalin groups were adjusted near 

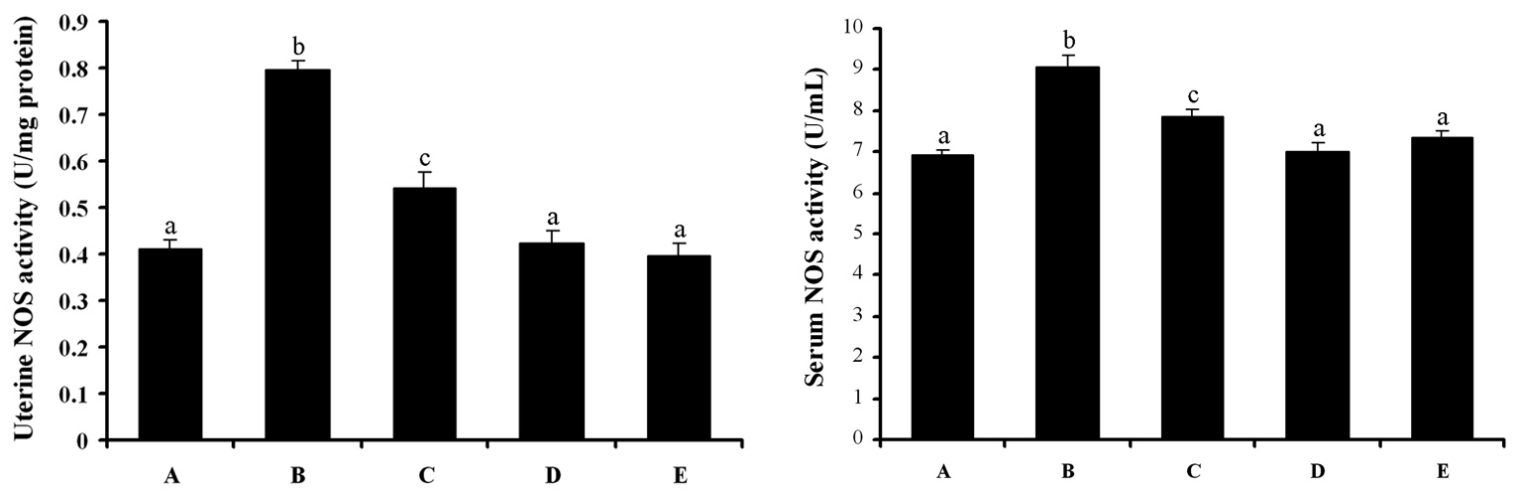

Figure 2. The uterine and serum NOS activity in different treatments mice $(n=8)$. Values of NOS activity were expressed as $\mathrm{U} / \mathrm{mg}$ protein or $\mathrm{U} / \mathrm{ml}$. Significant differences $(p<0.05)$ among all the groups were determined by ANOVA and are indicated by different letters.
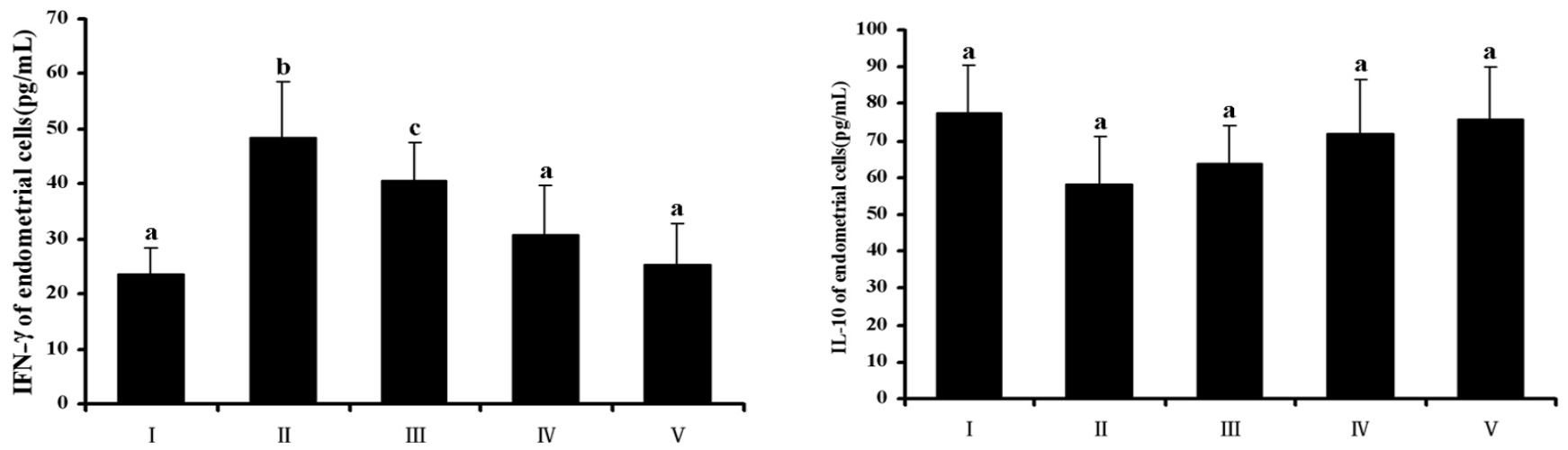

Figure 3. The IFN- $\mathbf{y}$ and IL-10 levels of endometrial cells culture supernatants at $48 \mathrm{~h}$ in different treatments. Significant differences $(p<0.05)$ among all the groups were determined by ANOVA and are indicated by different letters.

to control level, and they were different in the low-dose baicalin group. The trend of serum NOS activity was similar to the uterine.

\section{LPS increases IFN-y level of endometrial cells culture supernatant and baicalin's protective effects, but do not alters IL-10 level}

In the study of endometrial cell culture, the IFN-y level of LPS interference group was significantly increased when compared with control. It was significantly decreased by baicalin $(400$ and $800 \mu \mathrm{g} / \mathrm{ml})$ when compared with LPS interference group. After an increase in the concentration of baicalin, the IFN-y level was adjusted near to the control level (Figure 3).

The IL-10 level in the study of endometrial cell culture was not influenced significantly by LPS and baicalin treatment. Baicalin treatment increased IL-10 content a little; however, there was no difference when compared with control and LPS interference groups $(p>0.05)$. However, the ratio of IFN- $/ / \mathrm{LL}-10$ was significantly increased with LPS inference, and it was decreased significantly with baicalin near to control level (Figure 3).

\section{LPS and baicalin alters the NOS activity in endometrial cells culture supernatant}

The NOS activity was significantly increased by LPS inference when compared with control. However, baicalin normalized the NOS activity near to control level in the two higher concentration groups. Although, the baicalin with low concentration can decrease the NOS activity, there was no difference when compared with LPS interference group. The results herein indicated that baicalin down-regulates NO production from endometrial cells (Figure 4).

\section{DISCUSSION}

Pregnancy is a complex, sophisticated physiological process. The immune balance at the maternal-fetal interface 


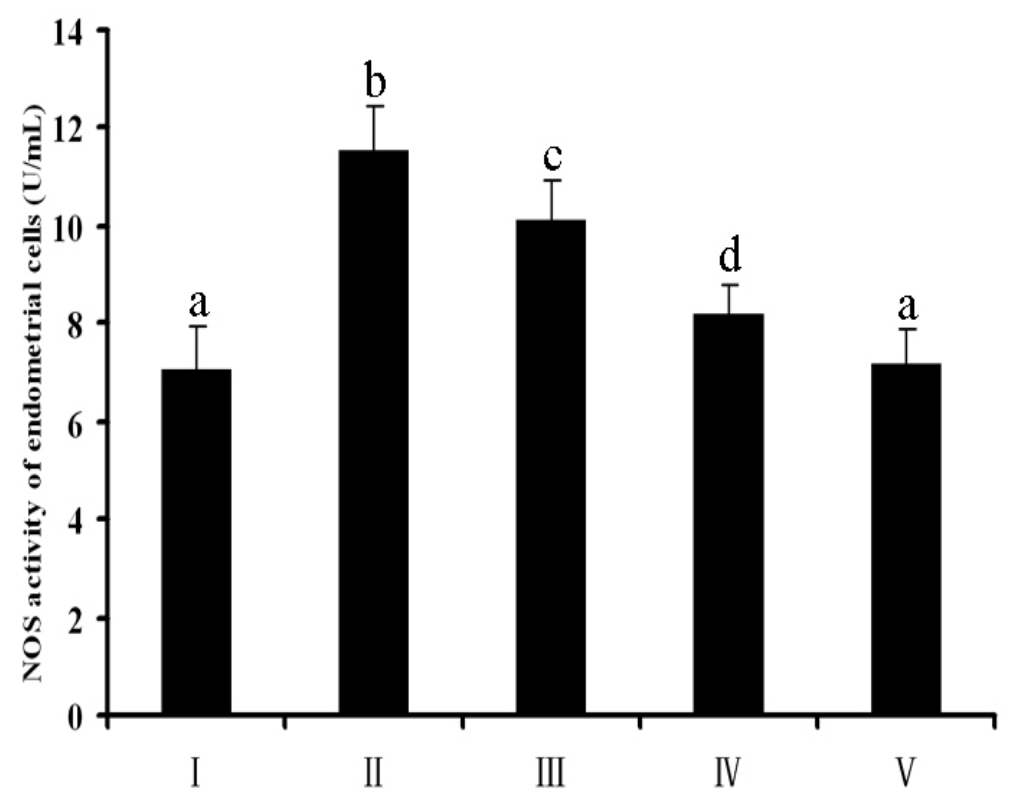

Figure 4. The NOS activity of endometrial cell culture supernatants at $48 \mathrm{~h}$ in different treatments. Values of NOS activity were expressed as $\mathrm{U} / \mathrm{ml}$. Significant differences $(p<0.05)$ among all the groups were determined by ANOVA and are indicated by different letters.

is necessary for the complicated pregnancy establishment and maintenance, especially $\mathrm{T}$ helper 1 (Th1)/Th2 cytokine balance. T lymphocytes play a key role in regulating and deviating immunological response by producing cytokines. There are two kinds of lymphocyte subpopulations, named Th1 and Th2 cells. Th1 cells produce mainly interleukins (IL) IL-1, IL-2, IL12, IL-15, IL-18, IFN- $y$ and TNF- $\alpha$ and Th2 cells are the source of IL-4, IL-5, IL-6, IL-10, IL-13, etc., (Wilczyński, 2005). The Th1/Th2 cytokine balance was destroyed when the pregnancy failure occurred, especially under abnormal situations, deviated towards Th1 activity demanding the IFN- $y, \mathrm{IL}-12$ and TNF- $\alpha$ (Zhong et al., 2008; Rosbottom et al., 2008; Wang et al., 2011).

Previous studies showed that endotoxin exposure contributed to the termination of pregnancy at any stage of gestation (Giri et al., 1990). However, the mechanism is not known clearly. The possible mechanism of LPSinduced abortion might be due to the secretion of tumor necrosis factor-alpha (TNF- $\alpha$ ) by triggered macrophages (MФ), which increased the cytotoxicity of uterine natural killer (NK) cells. Priming macrophages by interferons and activation by LPS resulted in the production of TNF (Gifford And Lohmann-Matthes, 1987). LPS is able to stimulate $\mathrm{T}$ lymphocytes to produce interferon-gamma (IFN-y) (Blanchardet al., 1986) and human peripheral blood mononuclear cells and splenocyte of mice to produce IFN- $y$. The Th1 cytokines over-expression in the uterus, such as IFN- $\gamma$ and TNF- $\alpha$, affects the precious immunological response at the feto-maternal interface at embryo implantation stage. Meanwhile, the balance of Th1/Th2 was altered towards to Th1 predominant which is detrimental to pregnancy and induced embryo loss (Clark et al, 2004). The serum TNF- $\alpha$, IL-1 $\alpha$, IL-6 levels and the receptor of $M \Phi$ induced by LPS were increased; however, there was no immunological response in fetal tissue, because LPS may not pass through the placentas and mainly affects the maternal immunologic tolerance (Rounioja et al., 2005). In addition, LPS is also recognized as the activator of nitric oxide (NO), and NO fulfills important functions during pregnancy and has a role in implantation, decidualization, vasodilatation and myometrial relaxation. However, the high concentration of NO has toxic effects as it is a free radical (Ogando et al., 2003).

Baicalin is a flavonoid compound purified from the medicinal plant and one of the major components of Chinese medicinal herb Huangqin ( $R$. scutellariae), which has an anti-abortive effect through inhibiting NK cell infiltration and regulating the cytokines at the maternalfetal interface (Zhong et al., 2002). Baicalin has been reported to possess anti-inflammatory and anti-viral activities. Baicalin inhibited the binding of a number of chemokines to human leukocytes or cells transfected to express specific chemokine receptors (Li et al., 2000). It has been shown that baicalin inhibited lipopolysaccharide-induced nitric oxide production and decreased inducible nitric oxide synthase gene expression 
in mouse macrophages (Nagaki et al., 2001) and inhibited the PGE2-induced aqueous flare elevation in pigmented rabbits.

Some studies have shown that Th1 cytokines, such as IFN- $\gamma$ and TNF- $\alpha$ are associated with infertility and abortion. Moreover, research about mice has shown that although, excessive levels of IFN- $y$ are deleterious for the establishment of pregnancy, uterine NK cell-derived IFN-y contributes to a normal pregnancy and facilitates pregnancy-induced artery remodeling (Ashkar et al., 2000). The IFN-y, as potential abortifacient, was significantly increased in LPS and parasite infection induced abortion (Rosbottom et al., 2008; Wang et al., 2011). In our study, the uterine IFN-y levels significantly increased in LPS treated mice and in LPS interference endometrial cells. Meanwhile, the average number of implanted embryo of LPS model group (B) was significantly less than other groups. These results are consistent with the previous reports and indicate that excess IFN- $y$ might be one of the main reasons of LPS induced mice embryo implantation failure. But IFN-y was normalized with middle and high dose baicalin. So, baicalin might protect the LPS-induced implantation failure through normalizing the Th1 cytokine (IFN- $\mathrm{y}$ ) level.

IL-10 was secreted by the Th2 cells and is a potent immune-regulating cytokine and inhibitor of inflammatory cytokine synthesis. IL-10 is a central regulator of the inflammatory response, acting to limit inflammationinduced tissue pathology by terminating monocyte and macrophage synthesis of TNF- $\alpha$ and an array of other proinflammatory cytokines and chemokines (Moore et al., 2001). Experiments in rodent models and humans implicate IL-10 in controlling inflammatory processes in pregnancy. IL-10 is expressed abundantly in the decidual and placental tissues in mice (Lin et al., 1993; Chaouat et al., 1999). The study showed that IL-10 modulated resistance to inflammatory stimuli by downregulating expression of proinflammatory cytokines TNF- $\alpha$, IL-6, IL$1 \mathrm{~A}$ and $\mathrm{IL}-12$, acting to protect against inflammationinduced pathology in the implantation site, in low-dose LPS induced IL-10-deficient mice fatal loss model (Robertson et al., 2007). In LPS induced mice abortion model, IL-10 level was significantly lower than control mice and the certain IL-10 was necessary to maintain normal pregnancy (Zhong et al., 2008). In the present study, IL-10 level decreased a little with LPS treatment in mice at implantation stage, but there was no significant difference when compared with the control group. It was not consistent that previous results, maybe the situation of LPS treatment at implantation stage was different to the pregnancy stage. The IL-10 levels of the middle and high dose baicalin groups were significantly increased. The ratio of IFN- $\mathrm{y} / \mathrm{IL}-10$ was significantly increased with LPS treatment, but it is adjusted to near control level plus baicalin $(0.5 \mathrm{mg})$ administration. In vitro experiment, the IL-10 level of culture supernatant did not alter significantly in LPS interference endometrial cells than control. The
IFN- $\mathrm{y} / \mathrm{LL}-10$ balance deviated towards IFN- $\gamma$ bias situation, and it is similar to the in vivo results. The excess IFN-y might be the reason for causing the implantation failure. However, baicalin normalized the IFN-y/LL-10 balance near to control level in the in vivo and in vitro studies in a dosage dependent manner. Baicalin has the protective effects on LPS-induced IFN$\mathrm{Y} / \mathrm{IL}-10$ changes in uterus and endometrial cells, normalizing the IFN-y/IL-10 balance to be beneficial to embryo implantation.

Nitric oxide (NO) is a multifunctional biomolecule that is produced by the enzyme nitric oxide synthase (NOS). Nitric oxide (NO) is an important factor in female reproductive processes, including ovulation, menstruation, implantation, pregnancy maintenance, labor and delivery (Chwalisz and Garfield, 2000), and also, has important roles during pregnancy in placental and uterine vessels and myometrial relaxation. High levels of NO are involved in antimicrobial and antitumor activities, and an elevation of NO can be proinflammatory (Weinberg, 1998). By contrast, low levels of NO are important for several physiological processes, including maintenance of smooth muscle tone, neurotransmission and modulation of apoptosis. The embryo implantation is unsuccessful with blocking NO production. The studies have shown that great amounts of NO and NOS might play a role in the pathogenesis of endometriosis (Wu et al., 2003), and NO might play an important role in the establishment of pregnancy in the rhesus monkey (Sengupta et al., 2005). NO production was increased in LPS-induced embryonic resorption mice, mainly due to the induction of expression of inducible nitric oxide synthase (iNOS) isoform in the decidua and uterus, and neuronal nitric oxide synthase (nNOS) isoform in the decidua (Ogando et al., 2003). NO is also important in early embryonic survival. However, excessive NO values from exogenous NO donors were found to be harmful to preimplanting and implanting mouse embryos (Barroso et al., 1998). In our study, the NO productions in the serum, uterus and endometrial cells were also increased significantly by LPS. All the change trends were similar between NO and IFN- $\gamma$ with the same treatment. The study reported that NOS may be induced by IFN-y or LPS (Purcell et al., 1999). The NOS activities of LPS treatment and low dose baicalin $(0.1 \mathrm{mg})$ groups were high. The LPS induced the excess IFN-Y secretion, and then, IFN- $y$ induced the NOS up-regulated (mainly iNOS) to produce excess NO. IFN-y and NO had the synergetic effect on inducing the damage of uterus and embryo and caused the embryo implantation failure. The result was consistent with Xie et al. (2002) report. However, the middle and high dose baicalins can normalize the IFN-y and NO level together and protect from embryo implantation failure. Meanwhile, the IL-10 might inhibit the NO and IFN- $y$ productions induced by LPS, and make the micro-environment of maternal-fetal interface beneficial for implantation. 


\section{Conclusion}

Conclusively, the present study has demonstrated that LPS produces the embryo implantation failure and that IFN- $/ / \mathrm{LL}-10$ balance and NO participate and play a key role in this process. IFN- $y$ and NO have synergetic effect on inducing embryo implantation failure. Baicalin, which is one of the major flavonoid components of Chinese traditional medicinal herb Huangqin ( $R$. scutellariae), has protective effects on LPS induced implantation failure through inhibiting IFN- $\gamma$, NO production of uterus and confirmed in endometrial cells. Meanwhile, baicalin increased the IL-10 level of mice uterus and adjusted the IFN- $/$ /L-10 balance in uterus and endometrial cells, normalizing the IFN- $\mathrm{Y} / \mathrm{LL}-10$ balance to be beneficial to embryo implantation. More studies need to be carried out to explain the mechanisms of the effects since there is a complex immune and endocrine network of regulation during embryo implantation and pregnancy.

\section{ACKNOWLEDGEMENTS}

This work was supported by grants from The National Natural Science Foundation of China (No. 30972208) and the Ministry of Education of China (No. 20101302110004).

\section{REFERENCES}

Ashkar AA, Di Santo JP, Croy BA (2000). Interferon-y contributes to initiation of uterine vascular modification, decidual integrity, and uterine natural killer cell maturation during normal murine pregnancy. J. Exp. Med., 192: 259-270.

Barroso RP, Osuamkpe C, Nagamani M, Yallampalli C (1998). Nitric oxide inhibits development of embryos and implantation in mice. Mole. Human Reprod., 4(5): 503-507.

Blanchard DK, Djeu JY, Klein TW (1986). Interferon-yinduction by lipopolysaccharide: dependence on interleukin 2 and macrophages. J. Immunol., 136(3): 963-970.

Chaouat G, Cayol V, Mairovitz V, Dubanchet S (1999). Localisation of the Th2 cytokines IL-3, IL-4, IL10 at the murine feto-maternal interface during pregnancy. In: Gupta SK (ed.), Reproductive Immunology. New Delhi: Narosa, 61-70.

Chatterjee S, Gangula PRR, Dong YL, Yallampalli C (1996). Immunocytochemical localization of nitric oxide synthase-III in reproductive organs of female rats during the estrus cycle. Histochem. J., 28: 715-723.

Clark DA, Manuel J, Lee L, Chaouat G, Gorczynski RM, Levy GA (2004). Ecology of danger-dependent cytokine-boosted spontaneous abortion in the CBA $\times$ DBA/2 mouse model. I. Synergistic effect of LPS and (TNF-alpha + IFN-gamma) on pregnancy loss. Am. J. Reprod. Immunol., 52(6): 370-378.

Chwalisz K, Garfield RE (2000). Role of nitric oxide in implantation and menstruation. Hum. Reprod., 15(3): 96-111.

de los Santos MJ, Mercader A, Galan A, Albert C, Romero JL, Pellicer A (2003). Implantation rates after two, three, or five days of embryo culture. Placenta, 24(Suppl. B): S13-S19.

Gifford GE, Lohmann-Matthes ML (1987). Gamma interferon priming of mouse and human macrophages for induction of tumor necrosis factor production by bacterial lipopolysaccharide, J. Natl. Cancer Institute, 78(1): 121-124.

Giri SN, Emau P, Cullor JS, Stabenfeldt GH, Bruss ML, Bondurant RH, Osburn BI (1990). Effects of endotoxin infusion on circulating levels of eicosanoids, progesterone, cortisol, glucose and lactic acid, and abortion in pregnant cows. Vet. Microbiol., 21(3): 211-231.

Kalinka J, Radwan M (2006). The impact of dydrogesterone supplementation on serum cytokine profile in women with threatened abortion. Am. J. Reprod. Immunol., 55(2): 115-121.

Knowles RG, Moncada S (2002). Nitric oxide synthase in mammals. Biochem., 298: 249-258.

Krasnow JS, Tollerud DJ, Naus G, DeLoia JA (1996). Endometrial Th2 Cytokines expression throughout the merstrual cycle and early pregnancy. Hum. Reprod., 11: 1747-1754.

Ledee-Bataille N, Lapree-Delage G, Taupin JL, Dubanchet S, Frydman $R$ and Chaouat $G(2002)$. Concentration of leukaemia inhibitory factor (LIF) in uterine flushing fluid is highly predictive of embryo implantation. Hum. Reprod., 17: 213-218.

Lessey BA, Damjanovich L, Coutifaris C, Castelbaum A, Albelda SM, Buck CA (1992). Integrin adhesion molecules in the human endometrium Correlation with the normal and abnormal menstrual cycle. J. Clin. Invest., 90: 188-195.

Li BQ, Fu T, Gong WH, Dunlop N, Kung H, Yan Y, Kang J, Wang JM (2000). The flavonoid baicalin exhibits anti-inflammatory activity by binding to Chemokines. Immunopharmacology, 49(3): 295-306.

Lin H, Mosmann TR, Guilbert L, Tuntipopipat S, Wegmann TG (1993). Synthesis of $\mathrm{T}$ helper 2-type cytokines at the maternal-fetal interface. J. Immunol., 151: 4562-4573.

Moncada S, Palmer RMJ, Higgs EA (1992). Nitric oxide: physiology, pathophysiology and pharmacology. Pharmacol. Rev., 43: 109-142.

Moore KW, de Waal Malefyt R, Coffman RL, O'Garra A (2001). Interleukin-10 and the interleukin-10 receptor. Annu Rev. Immunol., 19: 683-765.

Nagaki Y, Hayasaka S, Kadoi C, Nakamura N, Hayasaka Y(2001). Effects of Scutellariae radix extract and its components (baicalein, baicalin, and wogonin) on the experimental elevation of aqueous flare in pigmented Rabbits. Jpn J. Ophthalmol., 45(3): 216-220.

Ogando DG, Paz D, Cella M, Franchi AM (2003). The fundamental role of increased production of nitric oxide in lipopolysaccharide-induced embryonic resorption in mice. Reproduction, 125(1): 95-110.

Purcell TL, Given R, Chwalisz K, Garfield RE (1999). Nitric oxide synthase distribution during implantation in the mouse. Mol. Hum. Reprod., 5(5): 467-475.

Robertson SA, Care AS, Skinner RJ (2007). Interleukin 10 regulates inflammatory cytokine synthesis to protect against lipopolysaccharide-induced abortion and fetal growth restriction in mice. Biol. Reprod., 76(5): 738-748.

Rosbottom A, Gibney EH, Guy CS, Kipar A, Smith RF, Kaiser P, Trees AJ, Williams DJ (2008). Upregulation of cytokines is detected in the placentas of cattle infected with Neospora caninum and is more marked early in gestation when fetal death is observed. Infect. Immun., 76 (6): 2352-2361.

Rounioja S, Räsänen J, Ojaniemi M, Glumoff V, Autio-Harmainen H, Hallman M (2005). Mechanism of acute fetal cardiovascular depression after maternal inflammatory challenge in mouse. Am. J. Pathol., 166(6): 1585-1592.

Simon C, Moreno C, Remohi J, Pellicer A (1998). Cytokines and embryo implantation. J. Reprod. Immunol., 39: 117-131.

Simon C, Martin JC, Pellicer A (2000). Paracrine regulators of implantation. Baillieres Best Pract. Res. Clin. Obstet. Gynaecol., 14: 815-826.

Sengupta J, Dhawan L, Lalitkumar PG (2005). Nitric oxide in blastocyst implantation in the rhesus monkey. Reproduction, 130(3): 321-332.

Wang XD, Ma AT, Shi WY, Geng MY, Zhong XH, Zhao YT (2011). Quercetin and Bornyl Acetate Regulate T-Lymphocyte Subsets and INF-y/IL-4 Ratio In Utero in Pregnant Mice. Evidence Based Complement Alternative Med., p. 745262.

Weinberg JB (1998). Nitric oxide production and nitric oxide synthase type 2 expression by human mononuclear phagocytes: Rev. Mol. Med., 4: 557-591.

Wilczyński JR (2005). Th1/Th2 cytokines balance-yin and yang of reproductive immunology. Eur. J. Obstet. Gynecol. Reprod. Biol., 122(2): 136-143.

Wu MY, Chao KH, Yang JH, Lee TH, Yang YS, Ho HN (2003). Nitric oxide synthase is increased in endometrial tissue of women with endometriosis. Hum. Reprod., 12(18): 2668-2671.

Xie QF, Deng ZP, Sun BG (2002). Research on the role of NO in early 
embryo resorption in mice. Chinese J. Vet. Sci. (in Chinese), 22(1): 90-93.

Yallampalli C, Dong YL, Gangula P, Fang R (1998). Role and regulation of nitric oxide in the uterus during pregnancy and parturition. J. Soc. Gynecol. Invest., 5: 58-67.

Zhang XS, Zhong XH (2010). Effects of Scutellaria baicalens is and Atracty lodes macrocep halaon IFN- $y$, IL-10 and NO Contents in Mice of Embryo Implantation. Progress Vet. Med., 31(6): 80-84.

Zhong XH, Zhou ZX, Li TS, Wang EQ, Shi WY, Chu SM (2002). Antiabortive effects of Radix scutellariae and Rhizoma atractylodis in mice. Am. J. Chinese Med., 30(1): 109-117.
Zhong XH, Shi WY, Ma AT, Gong XC, Zhai XH, Zhang T, Wang XD (2008). Effects of Radix scutellariae and Rhizoma atractylodis on LPS-induced abortion and the uterine IL-10 contents in mice. Am. J. Chinese Med., 36(1): 141-148. 